\title{
Dynamic Capabilities Enhancing Path: a Perspective on Human Resource Policy
}

\author{
Gao Yan ${ }^{1}$ and Enyang Gao, ${ }^{2}$ \\ ${ }^{1}$ Jilin Engineering Normal University, Changchun, Jilin, China; \\ ${ }^{2}$ Shenyang Jianzhu University, Shenyang, China \\ 2238552865@qq.com
}

*The corresponding author

Keywords: Human resource strategy; Dynamic capabilities; Mechanism of action; Upgrade path

\begin{abstract}
Dynamic Capabilities to maintain, enhance and create strategic premise of dynamic competitive advantage. From the perspective of human resource strategy, we proposed the establishment of a comprehensive system of recruitment and selection; training of human resources to establish a top-down development system; establish reasonable performance evaluation and compensation systems; good corporate human resources systems and establishing a flexible organization ways to enhance the dynamic capability of enterprises to maintain sustainable competitive advantage of enterprises.
\end{abstract}

\section{Introduction}

With the global economic integration trend, companies which market and industry environment is becoming increasingly complex, dynamic and uncertainty among enterprises increasingly fierce competition, competitive enterprises established in the steady state is the steady erosion of the environment, in the conventional market static equilibrium environment formed constantly being broken, to this end, enterprises urgently need to reconstruct changes in the market for dynamic capability system. This ability of the existing research system known as dynamic capabilities, it is the business integration, build, and internal reorganization and external resources to correct the operational ability to operate, thus the ability to dynamically adapt to rapidly changing complex environment.

\section{Ecosystem the Connotation of Dynamic Capabilities}

"Dynamic Capabilities and Strategic Management," a paper presented a framework of dynamic capabilities. Articles in the "dynamic capabilities" is defined as a capability that allows companies to reconfigure, change, transformation of existing core competencies and properly shaping the existing core capabilities and external resources, strategic and complementary assets and integration, in order to respond to external, time-sensitive, rapid changes in the competitive and innovative environment coexist imitation. Dynamic capabilities include three parts: process (processes), position (positions) and paths (paths). Which refers to the process management and organizational processes, including the three elements, coordination / integration (a static concept), learning (a dynamic concept) and recombination (transformation concept); position refers to the current point in time enterprise business assets owned by difficult trading, such as a specific technology assets, complementary assets, financial assets and geographic location; path is the path dependence, namely the direction of a business can now choose depends on its current position and previous development path.

The Structural Dimensions of Dynamic Capabilities. Discussion on the organizational structure of dynamic capabilities, has been the focus of research in this area, there are different 
views of different scholars. But in the discussion of organizational structures in most dynamic capabilities emphasize the aspects of the identification of opportunities, respond to change, reorganization of resources and utilization.

The Ability to Identify Opportunities. Identify opportunities in the market is to identify suitable opportunities, including products or services. Global competitive environment, customer demand, technological opportunity, competitors in the high-speed action and so always changing, so grasp the opportunity, it is a scanning system, creating, learning and translation process, but also need to feature corresponding to $\mathrm{R} \& \mathrm{D}$ activities as a supplement.

The Ability to Respond to Changes in. The so-called capability is the ability to change in response to dynamic enterprises in the competitive environment in the face of competition requires numerous manifested. When the change is difficult to predict the dynamic and complex environment occurs, have the ability to respond to changes in the enterprise system can scan the environment, evaluation of competitors and market before competitors quickly complete the reconstruction and transformation of the business model of resources.

The Ability to Integrate Resources. Ability to integrate resources manager is to identify, capacity development and the use of specific resources to purchase or build competitive advantage through the integration of resources, companies can create a specific value. The ability to increase the conversion of resources to the business environment, adaptability, thus reducing the cost of trial and error; reconstruction resources reduced sunk costs, but also makes the enterprise in the face of turbulent environment may exhibit greater resource flexibility.

\section{Human Resource Management Strategy for The mechanism of Dynamic Capabilities}

The basic functions of micro-enterprise human resource management can be summarized configured as human resources, human resources training and development, human resources, salaries, building human resources systems into four categories. Human resources policies will affect both employees' knowledge and skills, also to affect the work motivation and work behavior of employees through their effects on aspects of staff and values, and employee skills and behaviors are dynamic organization with the ability to maintain a close inseparable link.

Staff Skills and Organizational Behavior Is the Basis of Dynamic Capabilities. The ability to organize the employees by organizational capacity transformed from the continuation and development and in the efforts of staff. If the enterprises to effectively improve and enhance the knowledge and skills of employees through human resource strategy, organizational behavior and making it consistent, then accumulate at a higher level of organizational procedures and organizational routines are formed, the organizational skills also formed. And this is achieved through human resource strategy based on organizational skills of employees is difficult to imitate by other companies. Dynamic capabilities as an important part of organizational capacity of the organization, which also depends on the formation and development of staff skills and behaviors to enhance employees' skills will inevitably bring to enhance the dynamic capability, and the consistency of staff and organizational behavior help build the organization. "flexible ", so as to enhance the dynamic capability of the organization. Therefore, staff skills and organizational behavior is the basis for building dynamic capabilities, directly on the organizational dynamic capability.

Human Resource Allocation and Human Resource. training and development of staff skills and behavior has a direct effect HR configuration includes recruitment and selection and job turnover: recruitment and selection is designed to refer to employ those candidates who have the skills and behaviors that meet specific needs of the work environment, the behavior of the organization will determine the level of quality for new employees; job turnover refers to the organization within the deployment of service personnel, promotion, demotion, rotation, etc., this behavior will have a direct impact on employee behavior. Human resource training and development can effectively improve staff quality, first improve staff skills, knowledge and ability to work-related and effectively motivate employees; secondly, training staff also improved its work itself and workplace satisfaction, further affect their behavior. Therefore, human resources development and 
training of human resources through a direct effect on employees' skills and behaviors affect the organization's dynamic capabilities.

Compensation and Human Resources Building Human Resources Systems Have an Indirect Impact On Employees' Skills and Behaviors. Salary incentives in corporate human resource strategy played a role in two aspects: one external one employee performance enhancement, first, to maintain internal equity. These two aspects of the role will affect employees through the psychological, and thus have an indirect effect on employee skills and behaviors. Human resources system construction related to all aspects of the organization, whether it is design or organization staff involvement, staff can implement effective incentive and restraint, on the one hand you can pass to an organization wants employees conduct their performance, on the other hand you can pass the values of the organization and specification, enabling them to obtain intrinsic motivation, and thus reflected in their behavior. Building human resources systems through the same psychological changes affecting staff employee skills and behaviors. Therefore, compensation and human resources human resources system construction through indirect effects on employees' skills and behaviors affect the organization's dynamic capabilities. .

\section{The Dynamic Capability of Human Resources Based on Business Strategies to Enhance the Way}

Establish a Comprehensive Recruitment and Selection System. Recruitment is the way enterprises to absorb external human resources major. Since the original enterprise employees work in the presence of path dependence, potentially hinder the formation of dynamic capabilities. Therefore, the introduction of new members of the soil through recruitment and selection, companies can not only update their own talent pool, improve soil quality and staff, and can effectively overcome the existing path dependence. Complete Recruitment system not only from the point of view of the candidates the skills level of education, skills, experience a soil for by other aspects of the measure for selection to those that meet business needs, with specific skills and different from the existing staff members skilled staff, improve the existing advantages of enterprise knowledge and technology, promote the diversification of employees skills; but also to candidates for a character, mentality, values and other aspects of evaluation, those on the high degree of recognition of the corporate culture, and business members agreed development goals absorbed into the soil to the enterprise, to enhance the dynamic capability of enterprises have a great role in promoting.

The Establishment of a Top-down Training of Human Resources Development System. Dynamic Capabilities in a changing environment in the formation. Therefore, the business environment, the ability to identify opportunities in the dynamic capabilities ability to identify, respond to changes in production and development capacity of the two dimensions have an important impact. Establish a top-down training of human resources development system, its purpose is to continuously upgrade the skills of existing internal staff, internal staff to develop new skills in order to enhance their adaptability to the environment. Especially for business executives in a training development system can help improve their self-confidence and common sense to update itself Knowledge base so under conditions relevant policy and legal environment changes, can quickly insight into the environment, and make the correct and feasible adjustment programs and advocate for change corporate strategy, organizational structure and mode of operation.

Establish A Reasonable Performance Evaluation and Compensation Systems. Objective and fair performance appraisal system for enterprise employees can form an effective incentive, thus affecting members soil behavior. Good combination of salary system and performance appraisal, performance appraisal system is to strengthen the incentive effects. Soil corporate staff performance appraisal should not only stay in the member territories technical level, staff attitude, mental activity and other spiritual evaluation should also be possible to use quantitative criteria to measure, in order to ensure that employees and organizational behavior of enterprises consistency, in order to enhance the integration of enterprise resource allocation and the scope and the speed right. Furthermore, it should establish a rapid evaluation of the results of the feedback system, take different measures motivation, communication and so on for the correct evaluation of the results of the different 
members of the soil to adjust the behavior of members of the soil, and the evaluation results combined with enterprises' salary system, to further strengthen the performance evaluation of employee behavior incentives.

Do the Human Resources System Construction Enterprises, The Establishment of Flexible Organization. As an important part of the strategy of flexible organizational dynamic capabilities for enterprises to adjust itself to adapt to the rapidly changing external environment, enhance performance and promote the survival and development are of great significance. Establish flexible organization from the following aspects:

To enhance the fit employee values and organizational culture. Enhance the value of employees can be through training, we can also organize daily activities for employees to strengthen, through extensive publicity to staff the organization's goals, vision, organizational values, and to strengthen communication with employees to achieve this goal.

Establish a flat organization. Flat organization in favor of democratization organizational communication and improve decision-making, enhanced dynamic adaptive organization. In organizational design should broaden the range of management, reducing layers of management, the establishment of the pass issued communication system.

Support employee participation. In order to respond more quickly and effectively to changes in the environment, employee participation should be strongly supported by managers. Managers should also by transforming their role, that is, from the control into the guide, cooperation, a large number of exceptions to the decentralization of decision-making staff. So that employees can make both work-related management decisions to develop their skills, but also the flexibility to adopt different measures in the face of specific problems, all kinds of exceptions processing appearing.

\section{Conclusion}

HR strategy of the enterprise dynamic ability has a direct or indirect role, we must, through the establishment of a comprehensive system of recruitment and selection; training of human resources to establish a top-down development system; establish reasonable performance evaluation and compensation systems; good corporate human resources system construction, the establishment of a flexible organization and other methods to enhance the dynamic capability of enterprises to maintain sustainable competitive advantage.

\section{References}

[1] Chen yuang, The ultimate goal of the enterprise information: build health [J]. Intelligence magazine,2007,(6):108-110.

[2] BrueeW. Managingthein formation eeology: Aeollaborative approaeh To information teechnologymanagement.QualityProgress, 1997,579

[3] Erik Assadourian. Global Economic Growth Continues at Expense of Ecological

[4] SA.L.Eryomin. Ecology and Society's Development[C]. 1st International Conference, Saint-Peter burg, Center of IAESVS, 1995:238-239. 